\title{
A New Method to Handle Conflict when Combining Evidences Using Entropy Function and Evidence Angle with an Effective Application in Fault Diagnosis
}

\author{
Lei Chen, ${ }^{1,2}$ Ling Diao, ${ }^{3}$ and Jun Sang $\mathbb{C}^{1}$ \\ ${ }^{1}$ School of Big Data \& Software Engineering, Chongqing University, Chongqing 401331, China \\ ${ }^{2}$ Dean's Office, Chongqing Aerospace Polytechnic, Chongqing 400021, China \\ ${ }^{3}$ Department of Computer Engineering, Chongqing Aerospace Polytechnic, Chongqing 400021, China
}

Correspondence should be addressed to Jun Sang; jsang@cqu.edu.cn

Received 7 April 2020; Revised 14 July 2020; Accepted 18 August 2020; Published 22 October 2020

Academic Editor: Filippo Cacace

Copyright (c) 2020 Lei Chen et al. This is an open access article distributed under the Creative Commons Attribution License, which permits unrestricted use, distribution, and reproduction in any medium, provided the original work is properly cited.

Addressing the problem of fusing highly conflicting evidences in Dempster-Shafer theory is one of the most necessary, important, and difficult research directions all the time, and so far we have published two papers related to it. In this paper, another novel method to handle conflict when combining evidences is proposed, where evidence distance, evidence angle, and improved entropy function, three key tools, are used for constructing the final weight of each body of evidence. This newly proposed approach mainly consists of three steps: firstly, both evidence distance and evidence angle determine the initial weight together; secondly, making use of the improved entropy modifies the initial weight to get the final weight; lastly, the classical D-S combination rule will be applied to obtain final fusion results. Still a classical numeric example and a real fault diagnosis application both demonstrate its effectiveness and efficiency, and compared with other current popular methods including two of our previous works, this new approach can converge fast and reduce most uncertainty of decision-making when fusing highly conflicting evidences.

\section{Introduction}

In practical applications, most information is collected by sensors. Because of the complexity of the target, the data provided by only one sensor may not be comprehensive enough to reflect the fact. Therefore multisensors are needed to produce more data for data fusion. However, the information derived from multiple sensors could be uncertain and even sometimes they are in conflict, which will confuse decision-makers [1-5], and so analyzing and handling this multisource uncertain information comprehensively need paying much attention. Dempster-Shafer theory of evidence (D-S theory) is such a powerful information fusion tool to address problems about uncertain information in intelligent systems [6-9]. In 1967, D-S theory was first presented by Dempster [1] and then improved by Shafer [10], Dempster's student. Actually, D-S theory is a generalization of traditional probability theory. Now, there are more and more fields where D-S theory is applied successfully, such as target recognition [11-14], reliability analysis [15-17], decisionmaking $[9,18-20]$, supplier selection [21-23], and optimization under uncertain environment [24-26].

Although D-S theory has lots of advantages as a tool of dealing with uncertainty, the counter-intuitive results are often generated when combining highly conflicting evidences with Dempster's combination rule [27-30]. So far, plenty of researchers have studied this problem and proposed different kinds of solutions. To sum up, these solutions can be divided into two main directions. One is to preprocess the bodies of evidence (BOEs) $[31,32]$ and the other is to modify the Dempster's combination rule $[33,34]$. Originally, Shafer used the coefficient $k$ to measure the conflict degree between the evidences [10], and then, in 2000 [33], Murphy presented a simple averaging approach, where the arithmetic average of $n$ evidences is calculated and then the Dempster's combination rule is utilized for fusion. 
However, this idea is not reasonable at all in practice because all BOEs can not be seen equally important. In 2004, Deng et al. [34] defined the dissimilarity measure to represent the conflict and proposed a weighed combination rule, where the weight average of the masses will be combined for $n-1$ times. Next, Han modified Deng's method using the Jousselme distance and information entropy. Later on, we also proposed two ideas to cope with this critical problem $[35,36]$.

In this paper, another novel weighted evidence combination rule is presented by us. In this time, the evidence angle is added to help us construct a more reasonable weight of each BOE. This newly proposed approach is composed of three key steps: firstly, both evidence distance and evidence angle determine the initial weight together; secondly, making use of the improved entropy modifies the initial weight to get final weight; lastly, the classical D-S combination rule will be applied to obtain final fusion results. Still a classical numeric example and a real fault diagnosis application both demonstrate the effectiveness and efficiency of our approach, and the comparison with other current popular methods including two of our previous works is also provided to show that our approach can converge fast and reduce most uncertainty of decisions when handling highly conflicting evidences.

The remaining paper is organized as follows: Section 2 starts with preliminaries about D-S theory, evidence distance, evidence angle, and improved belief entropy; Section 3 shows the proposed method in detail; Sections 4 and 5 give a numerical example and an application in faulty diagnosis to demonstrate its effectiveness and the efficiency; In the end, a short conclusion is made.

\section{Preliminaries}

In this section, some preliminaries are briefly introduced.

2.1. Basics of Evidence Theory. Dempster-Shafer theory of evidence (D-S theory) is made use of to cope with uncertainty information as an efficient mathematical model in intelligent systems [1]. In 1967, the definition of D-S theory was proposed by Dempster and then Shafer developed this theory in 1976 [10].

Let $\Omega$ be a nonempty finite set and $2^{\Omega}$ be the set of all subsets of $\Omega$, denoted $2^{\Omega}=\left\{\varnothing,\left\{\theta_{1}\right\},\left\{\theta_{2}\right\}, \ldots,\left\{\theta_{n}\right\}\right.$, $\left.\left\{\theta_{1}, \theta_{2}\right\}, \ldots,\left\{\theta_{1}, \theta_{2}, \ldots, \theta_{n}\right\}\right\}$. In D-S theory [10], a basic probability assignment (BPA) is a mapping $2^{\Omega} \longrightarrow$ $[0,1]$ that satisfies the following equations:

$$
\begin{aligned}
\sum_{\theta \subseteq \Omega} m(\theta) & =1, \\
m(\varnothing) & =0 .
\end{aligned}
$$

If $m(\theta)>0, \theta$ is called a focal element, and the set of all the focal elements is called one body of evidences(BOE). When there are more than one independent BOE, Dempster's combination rule, equation (3) can be used to combine these evidences:

$$
m(\theta)=\frac{\sum_{\theta_{1}, \theta_{2} \subseteq \Omega, \theta_{1} \cap \theta_{2}=m} m_{1}\left(\theta_{1}\right) m_{2}\left(\theta_{2}\right)}{1-K},
$$

where $K=\sum_{\theta_{1} \cap \theta_{2}=\varnothing} m_{1}\left(\theta_{1}\right) m_{2}\left(\theta_{2}\right)$ stands for the conflict degree, also called normalization constant. What's noted is that the combination rule above makes sense only when the conflict degree $m_{\oplus}(\varnothing) \neq 1$; otherwise, the rule is not meaningful. Here, we give a specific example about combination rule and show the corresponding results in Table 1.

Example 1. Suppose that the frame of discernment $=$ $\left\{\theta_{1}, \theta_{2}, \theta_{3}\right\}$ is complete and there are two BOEs listed as follows:

$$
\begin{aligned}
& \mathrm{BOE}_{1}: m_{1}\left(\theta_{1}\right)=0.5, m_{1}\left(\theta_{2}\right)=0.2, m_{1}\left(\theta_{3}\right)=0.3, \\
& \mathrm{BOE}_{2}: m_{2}\left(\theta_{1}\right)=0.6, m_{2}\left(\theta_{2}\right)=0.2, m_{2}\left(\theta_{3}\right)=0.2 .
\end{aligned}
$$

In the frame of discernment $\Omega$, there are two BOEs, $m_{1}$ and $m_{2}$. When $m_{1}$ and $m_{2}$ are both reliable, to generate a new BPA, we can apply the following conjunctive rule [37], denoted equation (5). When only one of them is totally reliable and we are not sure about another one, we should apply the disjunctive combination rule to obtain a new BPA as equation (6).

$$
\begin{array}{ll}
m_{\sqcap}(\theta)=\sum_{\theta_{1}, \theta_{2} \subseteq 2^{\Omega}, \theta_{1} \cap \theta_{2}=\theta} m_{1}\left(\theta_{1}\right) m_{2}\left(\theta_{2}\right), & \forall \theta \subseteq \Omega, \\
m_{\sqcup}(\theta)=\sum_{\theta_{1}, \theta_{2} \subseteq 2^{\Omega}, \theta_{1} \cap \theta_{2}=\theta} m_{1}\left(\theta_{1}\right) m_{2}\left(\theta_{2}\right), & \forall \theta \subseteq \Omega .
\end{array}
$$

Given a proposition $\theta_{1} \in 2^{\Omega}$, the belief function of $\theta_{1}$, denoted $\operatorname{Bel}\left(\theta_{1}\right)$, is defined in equation (7), which represents the total belief that the object is in $\theta_{1}$. The plausibility function of $\theta_{1}, \operatorname{Pl}\left(\theta_{1}\right)$ is defined in equation (8), which measures the total belief that can move into $\theta_{1}$. In D-S theory, $\operatorname{Bel}\left(\theta_{1}\right)$ and $\operatorname{Pl}\left(\theta_{1}\right)$ are called lower bound function and upper bound function, respectively, denoted $\left[\operatorname{Bel}\left(\theta_{1}\right), \operatorname{Pl}\left(\theta_{1}\right)\right]$. For any proposition, $\operatorname{Bel}\left(\theta_{1}\right)$ and $\operatorname{Pl}\left(\theta_{1}\right)$ satisfy the following relations of equations (9) and (10).

$$
\operatorname{Bel}\left(\theta_{1}\right)=\sum_{\theta_{2} \subseteq \theta_{1}} m\left(\theta_{2}\right)
$$

The plausibility function of $\mathrm{A}$, denoted $\mathrm{Pl}(\mathrm{A})$, is defined as follow:

$$
\begin{aligned}
& \operatorname{Pl}\left(\theta_{1}\right)=\sum_{\theta_{1} \cap \theta_{2} \neq \varnothing} m\left(\theta_{2}\right), \\
& \operatorname{Pl}\left(\theta_{1}\right)=1-\operatorname{Bel}\left(\overline{\theta_{1}}\right), \\
& \operatorname{Pl}\left(\theta_{1}\right) \geq \operatorname{Bel}\left(\theta_{1}\right) .
\end{aligned}
$$

Here, we give an example about belief function and plausibility function and show its results in Table 2.

Example 2. Assuming $\Omega=\left\{\theta_{1}, \theta_{2}, \theta_{3}\right\}$, a BPA is given that $m\left(\left\{\theta_{1}\right\}\right)=0.4, \quad m\left(\left\{\theta_{1}, \theta_{2}\right\}\right)=0.3, \quad m\left(\left\{\theta_{2}, \theta_{3}\right\}\right)=0.2$, and $m\left(\left\{\theta_{1}, \theta_{2}, \theta_{3}\right\}\right)=0.1$. 
TABLE 1: An example of the combination rule.

\begin{tabular}{lccc}
\hline BOEs & $m\left(\theta_{1}\right)$ & $m\left(\theta_{2}\right)$ & $m\left(\theta_{3}\right)$ \\
\hline BOE $_{1}$ & 0.5 & 0.2 & 0.3 \\
BOE $_{2}$ & 0.6 & 0.2 & 0.2 \\
Combined results & 0.75 & 0.1 & 0.15 \\
\hline
\end{tabular}

TABle 2: An example of Bel and Pl.

\begin{tabular}{lccccccc}
\hline Function & $\left\{\theta_{1}\right\}$ & $\left\{\theta_{2}\right\}$ & $\left\{\theta_{3}\right\}$ & $\left\{\theta_{1}, \theta_{2}\right\}$ & $\left\{\theta_{1}, \theta_{3}\right\}$ & $\left\{\theta_{2}, \theta_{3}\right\}$ & $\left\{\theta_{1}, \theta_{2}, \theta_{3}\right\}$ \\
\hline$m$ & 0.4 & 0 & 0 & 0.3 & 0 & 0.2 & 0.1 \\
Bel & 0.4 & 0 & 0 & 0.7 & 0.4 & 0.2 & 1 \\
Pl & 0.8 & 0.6 & 0.3 & 1 & 1 & 0.9 & 1 \\
\hline
\end{tabular}

But the classical Dempster's combination rule is not always in force. When BOEs are in high conflict, anti-intuitive results will be generated [35, 38-40]. So far, plenty of researchers have studied this problem and proposed different kinds of solutions. To sum up, these solutions can be divided into two main directions. One is to preprocess the bodies of evidence (BOEs) $[31,32]$ and the other is to modify Dempster's combination rule [33, 34]. Smets's unnormalized combination rule [41], Dubois and Prade's disjunctive combination rule [42], and Yager's combination rule [31] belong to the last category. These three alternatives mentioned above are examined and they all proposed a general combination framework. As for the first category, originally, Shafer used the coefficient $k$ to measure the conflict degree between the evidences [2], and then, in 2000 [33], Murphy presented a simple averaging approach, where the arithmetic average of $n$ evidences is calculated and then Dempster's combination rule is utilized for fusion. However, this idea is not reasonable at all in practice because all BOEs cannot be seen equally important. In 2004, Deng et al. [34] defined the dissimilarity measure to represent the conflict and proposed a weighed combination rule, where the weight average of the masses will be combined for $n-1$ times. Next, Han modified Deng's method using the Jousselme distance and information entropy [43]. Later on, we also proposed two ideas to cope with this critical problem $[35,36]$.

2.2. Evidence Distance. With D-S theory applying widely, the study about the distance of evidence has attracted more and more interests $[44,45]$. The dissimilarity measure of evidence can represent the lack of similarity between two BOEs. Multitarget performance assessment is done, such as performance evaluation [46], reliability evaluation [47], conflict evidence combination [34], and target association [48]. Lots of methods of evidence distance are brought up as an appropriate measure of the difference, and several definitions on distance in evidence theory are also proposed, such as Jousselme's distance [49], Wen's cosine similarity [50], Smets's transferable belief model(TBM) global distance measure [48], and Sunberg's belief function distance metric [51]. Among those definitions on the distance of evidence, the most frequently-used is Jousselme's distance [49].

The Jousselme's distance [49] is identified on the basis of Cuzzolin's geometric interpretation of evidence theory [52].
The power set of the frame of discernment $2^{\Omega}$ is regarded as a $2^{N}$ - linear space and a distance and vectors are defined with the BPA as a particular case of vectors. Jousselme's distance is defined as

$$
d_{i j}=\sqrt{\frac{1}{2}\left(\vec{m}_{i}-\vec{m}_{j}\right)^{T} D\left(\vec{m}_{i}-\vec{m}_{j}\right)}
$$

where $m_{i}, m_{j}$ are two BPAs under the frame of discernment $\Omega$ and $D$ is a $2^{N} \times 2^{N}$ matrix. The element in $D$ is defined as $D\left(E_{1}, E_{2}\right)=\left(\left|E_{1} \cap E_{2}\right| /\left|E_{1} \cup E_{2}\right|\right), E_{1}, E_{2} \in P\left(2^{\Omega}\right) ;|\cdot|$ represents cardinality. This Jousselme's distance satisfies all four requirements (nonnegativity, nondegeneracy, symmetry, and triangle inequality) [49] of a strict distance metric. Jousselme's distance is an efficient tool to quantify the dissimilarity of two BOEs. An example of Jousselme's distance is shown below.

Example 3. Assume there are two $\mathrm{BOEs}, \mathrm{BOE}_{1}$ and $\mathrm{BOE}_{2}$.

$$
\begin{aligned}
& \mathrm{BOE}_{1}: m_{1}\left(\theta_{1}\right)=0.4, m_{1}\left(\theta_{2}\right)=0.2, m_{1}\left(\theta_{1}, \theta_{2}\right)=0.1, m_{1}\left(\theta_{1}, \theta_{2}, \theta_{3}\right)=0.3 \\
& \mathrm{BOE}_{2}: m_{2}\left(\theta_{1}\right)=0.5, m_{2}\left(\theta_{2}\right)=0.2, m_{2}\left(\theta_{1}, \theta_{2}\right)=0.1, m_{2}\left(\theta_{1}, \theta_{2}, \theta_{3}\right)=0.2
\end{aligned}
$$

The value inside the BOE vectors $\overrightarrow{m_{1}}$ and $\overrightarrow{m_{2}}$ and the distance matrix $D$ are given by

$$
\begin{aligned}
& \overrightarrow{m_{1}}=\left(\begin{array}{c}
0.4 \\
0.2 \\
0.1 \\
0.3
\end{array}\right), \\
& \overrightarrow{m_{2}}=\left(\begin{array}{c}
0.5 \\
0.2 \\
0.1 \\
0.2
\end{array}\right) \\
& D=\left(\begin{array}{llll}
1 & 0 & \frac{1}{2} & \frac{1}{3} \\
0 & 1 & \frac{1}{2} & \frac{1}{3} \\
\frac{1}{2} & \frac{1}{2} & 1 & \frac{2}{3} \\
\frac{1}{3} & \frac{1}{3} & \frac{2}{3} & 1
\end{array}\right) .
\end{aligned}
$$

It follows 


$$
\begin{aligned}
\left(\overrightarrow{m_{1}}-\overrightarrow{m_{2}}\right)^{T} & =\left(\begin{array}{cccc}
-0.1 & 0 & 0 & 0.1
\end{array}\right), \\
\left(\overrightarrow{m_{1}}-\overrightarrow{m_{2}}\right) & =\left(\begin{array}{c}
-0.1 \\
0 \\
0 \\
0.1
\end{array}\right)
\end{aligned}
$$

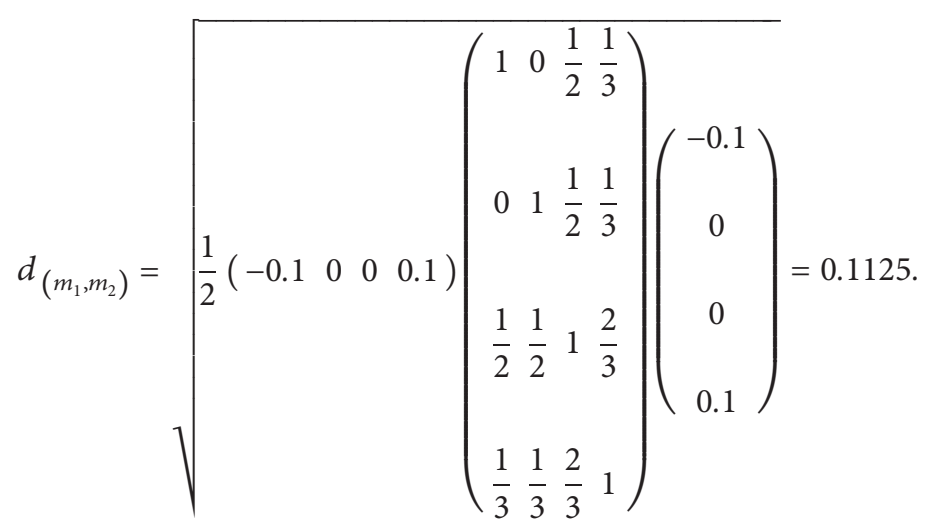

2.3. Evidence Angle. In this section, we describe the conflict or consistency degree between evidences from the perspective of geometry. Actually, each BOE can be regarded as a spatial vector and the angle of any two vectors can be used for characterizing the consistency between BOEs.

First, we introduce the pignistic vector angle. For a frame of discernment $\Omega$ consisting of $n$ elements, $2^{\Omega}$ is the set of all subsets of $\Omega$, and it can be denoted as $2^{\Omega}=\left\{\varnothing,\left\{\theta_{1}\right\}\right.$, $\left.\left\{\theta_{2}\right\}, \ldots,\left\{\theta_{n}\right\},\left\{\theta_{1}, \theta_{2}\right\}, \ldots,\left\{\theta_{1}, \theta_{2}, \ldots, \theta_{n}\right\}\right\}$. In order to correspond to the dimension of space vector, we need to transform the BPA vector into an $\mathrm{n}$-dimensional vector, where the converted $n$-dimensional vector can be obtained from the pignistic probability function.

Suppose $\mathrm{m}\left(\theta_{1}\right)$ is a BPA in the frame of discernment $\Omega$, then the pignistic probability transformation function $\operatorname{Bet} P_{m}\left(\theta_{1}\right)$ is defined in the following equation:

$$
\operatorname{Bet} P_{m}\left(\theta_{1}\right)=\sum_{\theta_{1} \subseteq \Omega, \theta_{2} \subseteq \Omega} \frac{\left|\theta_{1} \cap \theta_{2}\right|}{\left|\theta_{2}\right|} \frac{m\left(\theta_{2}\right)}{1-m(\phi)}
$$

where $m(\phi) \neq 1$ and $\left|\theta_{1}\right|$ are the number of elements in $\theta_{1}$.

Then, we make use of the cosine value of the pignistic vector angle between two BOEs to measure their consistency degree, defined in equation (16). The larger the value of $\cos \left(m_{i}, m_{j}\right)$ is, the more consistent these two BOEs are.

$$
\cos \left(m_{i}, m_{j}\right)=\frac{\operatorname{Bet} P_{m_{i}} * \operatorname{Bet} P_{m_{j}}}{\left\|\operatorname{Bet} P_{m_{i}}\right\| *\left\|\operatorname{Bet} P_{m_{j}}\right\|}
$$

where $m(\phi) \neq 1$ and $\left|\theta_{1}\right|$ are the number of elements in $\theta_{1}$.

Example 4. Assume $\Omega=\left\{\theta_{1}, \theta_{2}, \theta_{3}\right\}$, and there are two following BOEs we collected. We want to compute the consistency degree between these two BOEs.

$$
\begin{aligned}
B O E_{1}: m_{1}\left(\theta_{1}\right) & =0.5, m_{1}\left(\theta_{2}\right)=0.1, m_{1}\left(\theta_{1}, \theta_{2}\right) \\
& =0.2, m_{1}\left(\theta_{1}, \theta_{2}, \theta_{3}\right)=0.2, \\
B O E_{2}: m_{2}\left(\theta_{1}\right) & =0.5, m_{2}\left(\theta_{2}\right)=0.2, \theta_{2}\left(\theta_{1}, \theta_{2}, \theta_{3}\right)=0.3 .
\end{aligned}
$$

First, apply equation (15) to transform these two BPAs to n-dimensional vectors.

For $\mathrm{BOE}_{1}, \operatorname{Bet} P_{m_{1}}\left(\theta_{1}\right)=0.5+(0.2 / 2)+(0.2 / 3)=0.6667$, $\operatorname{Bet} P_{m_{1}}\left(\theta_{2}\right)=0.1+(0.2 / 2)+(0.2 / 3)=0.2667$ and $\operatorname{Bet} P_{m_{1}}$ $\left(\theta_{3}\right)=(0.2 / 3)=0.0666 ; \quad$ For $\mathrm{BOE}_{2}, \quad \operatorname{Bet} P_{m_{2}}\left(\theta_{1}\right)=0.5+$ $(0.3 / 3)=0.6, \operatorname{Bet} P_{m_{2}}\left(\theta_{2}\right)=0.2+(0.3 / 3)=0.3$, and $\operatorname{Bet} P_{m_{2}}$ $\left(\theta_{3}\right)=(0.3 / 3)=0.1$.

Then, measure the consistency degree $\cos \left(m_{1}, m_{2}\right)$ between $\mathrm{BOE}_{1}$ and $\mathrm{BOE}_{2}$ based on equation (16).

$$
\cos \left(m_{1}, m_{2}\right)=\frac{\operatorname{Bet} P_{m_{1}}\left(\theta_{1}\right) * \operatorname{Bet} P_{m_{2}}\left(\theta_{1}\right)+\operatorname{Bet} P_{m_{1}}\left(\theta_{2}\right) * \operatorname{Bet} P_{m_{2}}\left(\theta_{2}\right)+\operatorname{Bet} P_{m_{1}}\left(\theta_{3}\right) * \operatorname{Bet} P_{m_{2}}\left(\theta_{3}\right)}{\sqrt{\operatorname{Bet} P_{m_{1}}\left(\theta_{1}\right)^{2}+\operatorname{Bet} P_{m_{1}}\left(\theta_{2}\right)^{2}+\operatorname{Bet} P_{m_{1}}\left(\theta_{3}\right)^{2}} * \sqrt{\operatorname{Bet} P_{m_{2}}\left(\theta_{1}\right)^{2}+\operatorname{Bet} P_{m_{2}}\left(\theta_{3}\right)^{2}+\operatorname{Bet} P_{m_{2}}\left(\theta_{3}\right)^{2}}}
$$


and so

$$
\cos \left(m_{1}, m_{2}\right)=\frac{0.6667 * 0.6+0.2667 * 0.3+0.0666 * 0.1}{0.7211 * 0.6782}=0.9951 .
$$

2.4. Improved Belief Entropy. Uncertainty is widespread in the universe [53-58]. If a probability assignment $p$ is provided, we can apply the following Shannon entropy [59] to measure its uncertainty.

$$
H=-\sum_{i} p_{i} \log _{2}\left(p_{i}\right)
$$

But if a BPA is given, there is no way to measure that uncertainty based on some other main entropies listed in Table 3.

As for such a reason, the Deng entropy [66] is presented to measure the uncertainty of BPA, which is a more significant tool to manage uncertainty than Shannon entropy [59]. The Deng entropy can deal with the uncertainty represented not only by BPA but also by probability distribution. In other words, the Deng entropy is the generalization of the Shannon entropy [6, 67-69].

The Deng entropy can be denoted as follows:

$$
E_{d}=-\sum_{i} m\left(F_{i}\right) \log _{2}\left(\frac{m\left(F_{i}\right)}{2\left|F_{i}\right|}-1\right)
$$

where $F_{i}$ is a proposition in mass function $m$ and $\left|F_{i}\right|$ is the cardinality of $F_{i}$. Especially, if the belief is only assigned to single elements, the Deng entropy equals with the Shannon entropy [66], denoted

$$
E_{d}=-\sum_{i} m\left(\theta_{i}\right) \log _{2}\left(\frac{m\left(\theta_{i}\right)}{2^{\left|\theta_{i}\right|}-1}\right)=-\sum_{i} m\left(\theta_{i}\right) \log _{2} m\left(\theta_{i}\right) .
$$

However, there is a big shortcoming of the Deng entropy that it can not effectively quantify the difference among different BOEs which are assigned by the same mass value.
For example, there are two BOEs, $\mathrm{BOE}_{1}$ and $\mathrm{BOE}_{2}$, as follows:

$$
\begin{aligned}
& \mathrm{BOE}_{1}: m_{1}\left(e_{1}, e_{2}\right)=0.4, m_{1}\left(e_{3}, e_{4}\right)=0.6, \\
& \mathrm{BOE}_{2}: m_{2}\left(e_{1}, e_{2}\right)=0.4, m_{2}\left(e_{2}, e_{3}\right)=0.6 .
\end{aligned}
$$

According to equation (16), the uncertainty measures with the Deng entropy are, respectively, $E_{d}\left(\mathrm{BOE}_{1}\right)=2.5559$ and $E_{d}\left(\mathrm{BOE}_{2}\right)=2.5559$. However, this result calculated by the Deng entropy is counter-intuitive, because though these two BOEs have the same mass value, S1 consists of four targets, denoted as $e_{1}, e_{2}, e_{3}$, and $e_{4}$, while S2 has only three possible targets, and intuitively, what is expected that S2 should have less uncertainty than S1. That means the entropy value of S1 should be bigger than that of S2. Therefore, the Deng entropy cannot quantify this difference and we propose one improved entropy function, which can finish this job very well.

The improved belief entropy is defined as follows:

$$
E_{i}=-\sum_{i} m\left(\theta_{i}\right) \log _{2}\left(\frac{m\left(\theta_{i}\right)}{2\left|\theta_{i}\right|} \frac{\left|\theta_{i}\right|}{|\theta|}\right),
$$

where $\left|\theta_{i}\right|$ denotes the cardinality of the focal element $\theta_{i},|\theta|$ is the total number of elements in this BOE, and $\left(\left|\theta_{i}\right| /|\theta|\right)$ is used to represent the uncertain information in a $\mathrm{BOE}$ that has been ignored by the Deng entropy.

Still for two BOEs, $\mathrm{BOE}_{1}$ and $\mathrm{BOE}_{2}$ above, we calculate the uncertainty by means of the newly proposed entropy function, and we can get $E_{i}\left(\mathrm{BOE}_{1}\right)=\left(-0.4 * \log _{2}\left(\left(0.4 / 2^{2}-\right.\right.\right.$ $1) *(2 / 4)))+\left(-0.6 * \log _{2}\left(\left(0.6 / 2^{2}-1\right) *(2 / 4)\right)\right)=3.5559$ and $E_{i}\left(\mathrm{BOE}_{2}\right)=\left(-0.4 * \log _{2}\left(\left(0.4 / 2^{2}-1\right) *(2 / 3)\right)\right)+(-0.6$ $\left.* \log _{2}\left(\left(0.6 / 2^{2}-1\right) *(2 / 3)\right)\right)=3.1409$. So, this improved belief entropy can effectively quantify the difference even if the same mass values are assigned on different BOEs.

An example of the comparison of the Deng entropy and improved entropy function is given and the results are seen in Table 4.

Example 5. Assuming $\Omega=\left\{e_{1}, e_{2}, e_{3}\right\}$,

$$
\begin{aligned}
& \mathrm{BOE}_{1}: m_{1}\left(e_{1}\right)=0.3, m_{1}\left(e_{1}, e_{2}\right)=0.2, m_{1}\left(e_{1}, e_{2}, e_{3}\right)=0.5, \\
& \mathrm{BOE}_{2}: m_{2}\left(e_{2}\right)=0.2, m_{2}\left(e_{2}, e_{3}\right)=0.4, m_{2}\left(e_{3}\right)=0.4, \\
& \mathrm{BOE}_{3}: m_{3}\left(e_{1}\right)=0.2, m_{3}\left(e_{2}\right)=0.2, m_{3}\left(e_{1}, e_{3}\right)=0.3, m_{3}\left(e_{1}, e_{2}, e_{3}\right)=0.3, \\
& \mathrm{BOE}_{4}: m_{4}\left(e_{1}\right)=(1 / 3), m_{4}\left(e_{2}\right)=(1 / 3), m_{4}\left(e_{3}\right)=(1 / 3), \\
& \mathrm{BOE}_{5}: m_{5}\left(e_{1}, e_{2}, e_{3}\right)=1 .
\end{aligned}
$$

\section{The Proposed Method}

The newly proposed method follows our previous works $[35,36]$. Suppose $n$ pieces of independent BOEs $m_{i}(i=1, \ldots, n)$ have been collected now. What we need to consider first is to preprocess these BOEs through the following equation:

$$
\operatorname{WAM}(m)=\sum_{i=1}^{n} w_{i} \times m_{i}
$$

where $w_{i}$ is the corresponding weight degree of BOE $m_{i}$ and WAM $(m)$, the short-writing of weighted average mass, represents the weighted average $\mathrm{BPA}$ of $n$ independent BOEs. 
TABLE 3: Some main entropies to measure uncertainty.

\begin{tabular}{lc}
\hline Proposer & Equation \\
\hline Dubois and Prade [60] & $N_{\mathrm{DP}}(m)=\sum_{A \subseteq X} m(A) \log (|A|)$ \\
Yager [61] & $C_{Y}(m)=-\sum A \subseteq F m(A) \log P l(A)$ \\
Klir and Ramer [62] & $C_{K R}(m)=-\sum A \subseteq F m(A) \log \sum_{B \subseteq F} m(B)(|A \cap B| /|B|)$ \\
George and Pal [63] & $C_{\mathrm{GP}}(m)=-\sum A \subseteq F m(A) \sum_{B \subseteq F} m(B)(1-(|A \cap B| /|A \cup B|))$ \\
Maluf [64] & $C_{M}(m)=-\sum A \subseteq F P l(A) \operatorname{logBel}(A)$ \\
Klir [65] & $C_{K}(\operatorname{Bel})=-(1 / c) \sum x \subseteq X \operatorname{Bel}(\{x\}) \operatorname{logBel}(\{x\})+\operatorname{Pl}(\{x\}) \operatorname{logPl}(\{x\}), c=\sum_{x \subseteq X}[\operatorname{Bel}(\{x\})+\operatorname{Pl}(\{x\})]$ \\
\hline
\end{tabular}

Table 4: An example of the Deng entropy.

\begin{tabular}{lcccrr}
\hline Source of evidence & $\mathrm{BOE}_{1}$ & $\mathrm{BOE}_{2}$ & $\mathrm{BOE}_{3}$ & $\mathrm{BOE}_{4}$ & $\mathrm{BOE}_{5}$ \\
\hline Deng entropy [66] & 3.2061 & 2.1559 & 3.2886 & 1.5850 & 2.8074 \\
Improved entropy function [36] & 3.7986 & 2.7560 & 4.0981 & 3.1699 & 2.8074 \\
\hline
\end{tabular}

Referring to Murphy's work [33], we need to combine WAM $(m)$ for $n-1$ times by using classical Dempster's rule and then get the final combined results. However, to find an appropriate weight degree $w_{i}$ is not an easy thing. There have been lots of works related to computing a weight of BOE, such as Deng's work [34]. Based on what have done before, another novel idea comes up. At first, we calculate an initial weight of BOE based on both the evidence distance and evidence angle. The evidence distance represents the dissimilarity between evidences, whereas the evidence angle describes the inconsistency among evidences. Then, our previously proposed improved entropy function is used for characterizing the uncertainty of BOE and further modifying the initial weight in order to get a more accurate and reasonable weight. The details of the newly proposed approach are shown below.

\subsection{Determining Initial Weight Using Pignistic Vector Angle} and Jousselme Distance. At the beginning, apply equations (11) and (16) to respectively calculate the evidence distance $d\left(m_{i}, m_{j}\right)$ and the cosine value of evidence angle $\cos \left(m_{i}, m_{j}\right)$ between any two BOEs $m_{i}, m_{j}(i, j=1,2, \ldots, n)$.

Followed by our previous works and other popular papers in evidence theory, the conflict degree can be used to weigh the evidence. Here, both evidence distance and evidence angle are used to characterize the degree of conflict between the evidences in order to capture the two main aspects that affect the evidence conflicts. The evidence distance describes the dissimilarity between the evidences, whereas the evidence angle represents the inconsistency among the evidences. These two measures are mutually complementary in a sense, and based on the introduction of the evidence distance and evidence angle in Section 2, the smaller the distance between two BOEs is, the more similar they are, and the bigger the cosine value of evidence angle, the more consistent these two BOEs are. Therefore, we construct the similarity measure $\operatorname{sim}\left(m_{i}, m_{j}\right)$ between $m_{i}$ and $m_{j}$ like the following equation:

$$
\operatorname{sim}\left(m_{i}, m_{j}\right)=\left(1-d\left(m_{i}, m_{j}\right)\right)\left(\cos \left(m_{i}, m_{j}\right)\right) \text {. }
$$

Then, the support degree of a BOE $m_{i}(i=1,2, \ldots, n)$ could be defined [34] based on the similarity measure mentioned above:

$$
\sup \left(m_{i}\right)=\frac{\sum_{j=1, j \neq i}^{n} \operatorname{sim}_{i j}}{n-1} .
$$

After normalizing the support degree of each BOE, we can get its own initial weight $i w\left(m_{i}\right)$, which is determined by both the evidence distance and evidence angle function, as shown in the following equation:

$$
i w\left(m_{i}\right)=\frac{\sup \left(m_{i}\right)}{\sum_{j=1}^{n} \sup \left(m_{j}\right)} .
$$

As you can see, this initial weight satisfies $\sum_{i=1}^{n} i w\left(m_{i}\right)=1$. What to do next is just to modify $i w\left(m_{i}\right)$ by means of our previously proposed improved belief entropy [36].

3.2. Computing Final Weight on the Basis of Improved Entropy Function. In accordance with our intuition, one BOE $m_{i}$ with higher support degree $\sup \left(m_{i}\right)$ or initial weight $i w\left(m_{i}\right)$ should have less uncertainty. In a similar fashion, the BOE owning more uncertainty must have lower sup $\left(m_{i}\right)$ or $i w\left(m_{i}\right)$. On the ground of the thinking above, we could modify the initial weight $i w\left(m_{i}\right)$ through the following steps:

Step 1: make use of our previously proposed improved entropy function in equation (24) to get uncertainty degree $u\left(m_{i}\right)$ of each BOE $m_{i}(i=1, \ldots, n)$, and then, normalizing $u\left(m_{i}\right)$ by using equation (30), the normalized uncertainty measure $u n\left(m_{i}\right)(i=1, \ldots, n)$ can be obtained.

$$
u n\left(m_{i}\right)=\frac{u\left(m_{i}\right)}{\sum_{i=1}^{n} u\left(m_{i}\right)}
$$

Step 2: after modifying the initial weight $i w\left(m_{i}\right)$ by taking advantage of equations (31) and (32), the final weight $f w\left(m_{i}\right)(i=1, \ldots, n)$ of each BOE will be obtained. 


$$
\begin{aligned}
m d w\left(m_{i}\right) & =i w\left(m_{i}\right) \times e^{\left(n-u n\left(m_{i}\right)\right)}, \\
f w\left(m_{i}\right) & =\frac{m \mathrm{~d} w\left(m_{i}\right)}{\sum_{i=1}^{n} m \mathrm{~d} w\left(m_{i}\right)} .
\end{aligned}
$$

Step 3: use equation (33) to compute the weighted averaged $\operatorname{BOE} \operatorname{WAM}(m)$. Finally, apply the classical Dempster's rule [1] to combine $\operatorname{WAM}(m)$ for $n-1$ times [33] and the final combined results will be obtained.

$$
\operatorname{WAM}(m)=\sum_{i=1}^{n}\left(f w\left(m_{i}\right) \times m_{i}\right)(i=1,2, \ldots, n) .
$$

\section{Experiment}

In this section, a classic numerical example is provided to show how to use the new proposed method step by step and meanwhile to demonstrate its efficiency and effectiveness.

Example 6. In a multisensor-based automatic target recognition system, suppose that the frame of discernment $\Omega=$ $\left\{\theta_{1}, \theta_{2}, \theta_{3}\right\}$ is complete and $\theta_{1}$ is the real target. The system collects the following five BOEs from five different sensors:

$$
\begin{aligned}
& \mathrm{BOE}_{1}: m_{1}\left(\theta_{1}\right)=0.41, m_{1}\left(\theta_{2}\right)=0.29, m_{1}\left(\theta_{3}\right)=0.3, \\
& \mathrm{BOE}_{2}: m_{2}\left(\theta_{1}\right)=0, m_{2}\left(\theta_{2}\right)=0.9, m_{2}\left(\theta_{3}\right)=0.1, \\
& \mathrm{BOE}_{3}: m_{3}\left(\theta_{1}\right)=0.58, m_{3}\left(\theta_{2}\right)=0.07, m_{3}\left(\theta_{1}, \theta_{3}\right)=0.35, \\
& \mathrm{BOE}_{4}: m_{4}\left(\theta_{1}\right)=0.55, m_{4}\left(\theta_{2}\right)=0.1, m_{4}\left(\theta_{1}, \theta_{3}\right)=0.35, \\
& \mathrm{BOE}_{5}: m_{5}\left(\theta_{1}\right)=0.6, m_{5}\left(\theta_{2}\right)=0.1, m_{5}\left(\theta_{1}, \theta_{3}\right)=0.3 .
\end{aligned}
$$

Before getting the similarity measure $\operatorname{sim}\left(m_{i}, m_{j}\right)(i, j=$ $1,2, \ldots, n)$ between $m_{i}$ and $m_{j}$, we need to calculate the evidence distance and the cosine value of evidence angle among BOEs. Tables 5 and 6 separately show the results of $d\left(m_{i}, m_{j}\right)$ and $\cos \left(m_{i}, m_{j}\right)$ on condition of different numbers of BOEs.

Then, the values of similarity degree $\operatorname{sim}\left(m_{i}, m_{j}\right)$ between any two BOEs could be obtained by means of equation (27), and the corresponding results are shown in Table 7.

Secondly, based on the calculated similarity degree and equation (28), the support degree of each BOE $\sup \left(m_{i}\right)(i=$ $1,2, \ldots, n)$ could be acquired, as listed in Table 8 . Then, normalizing the support measure $\sup \left(m_{i}\right)$, we will get its own initial weight $i w\left(m_{i}\right)$ under different conditions, as listed in Table 9.

Next, measure the uncertainty degree $u\left(m_{i}\right)(i=1, \ldots, n)$ of each BOE by the improved entropy function and then make a normalization of $u\left(m_{i}\right)$ to get $u n\left(m_{i}\right)$ by virtue of equations (29) and (30). Tables 10 and 11 show the results of uncertainty measure under the condition of specific number of BOEs.

After having its uncertainty degree of each BOE, we can apply (31) and (32) to modify the initial weight $i w\left(m_{i}\right)$ and then the final weight $f w\left(m_{i}\right)(i=1, \ldots, n)$ can be calculated well. The results of $f w\left(m_{i}\right)$ are shown in Table 12 .
At last, compute the weighted averaged BOE WAM $(m)$ listed in Table 13 and apply the classical Dempster's combination rule [1] to fuse WAM $(m)$ for $n-1$ times ( $n$ is the total number of BOEs) [33], and then. we can get the final combined results, as shown in Table 14.

In order to demonstrate the efficiency and effectiveness of this newly proposed method, we have made a clear comparison with other current mainly popular combination rules by calculating this numeric example. The comparison results are all shown in Table 15 and Figures 1-4.

As shown clearly in Table 15 and Figures 1-4, the classical Dempster's combination rule [1] cannot handle the fusion of conflicting evidence well and the counter-intuitive combination results will be produced, and with incremental BOEs, Murphy's simple averaging [33], Deng et al.'s weighted averaging [34], and our last work all can get relatively reasonable results, but all of them are inferior to that of our newly proposed method. Most importantly, when we only have limited number of BOEs, this new method can give more convincing results to decision-makers. Moreover, compared with these current mainly popular evidence combination rules, the convergence of ours is best. That is because both the evidence angle and evidence distance can characterize the relation between any two BOEs better and the improved entropy function helps modify the initial weight more efficiently and reasonably, and so the effect of "good" evidence is strengthened extremely and the effect of "bad" one is weakened largely in the final combined results.

\section{Application in Diagnosis Fault}

Similar to our previous work $[35,36]$, this newly proposed approach is also applied to fault diagnosis area, and the example we use here cites from that of our previous work $[35,36]$. Suppose there is a machine which has three gears $G_{1}, G_{2}$, and $G_{3}$, and the failure fault modes $F_{1}, F_{2}$, and $F_{3}$ are three kinds of failure fault modes corresponding to $G_{1}, G_{2}$, and $G_{3}$ and are collected as fault hypothesis set $\left\{F_{1}, F_{2}, F_{3}\right\}$. Besides, there are three various sensors, $s_{1}, s_{2}$, and $s_{3}$, from which the evidence set $m_{1}, m_{2}, m_{3}$ shown in Table 16 comes.

Here, still two kinds of sensor reliability will be considered. One is the static reliability $R_{i}^{s}=\mu_{i} \times \nu_{i}$ measured by the evidence sufficiency $\operatorname{suf}(\mu)$ and importance index $\operatorname{imp}(\nu)$, and the other is dynamic reliability $R_{i}^{d}$ measured based on the final weight $f w\left(m_{i}\right)$ proposed newly in this work. The final comprehensive reliability $R=R^{s} \times R^{d}$ is used for correcting the highly conflicting evidences, and after combining those evidences, a result with a higher accuracy and more belief will be obtained.

At the beginning, let us compute the static reliability $R^{s}$ of each BOE using the formula $R_{i}^{s}=\mu_{i} \times \nu_{i}$ and the corresponding results are shown in Table 17.The next step is to compute the dynamic reliability $R^{d}$ of each $\mathrm{BOE}, \mathrm{BOE}_{1}, \mathrm{BOE}_{2}$, and $\mathrm{BOE}_{3}$; that is, we need to get the final weight $f w\left(m_{i}\right)(i=$ $1,2,3)$ by means of our newly proposed method in Section 3.

To get $f w\left(m_{i}\right)$, what we need to do first is to calculate the evidence distance $d\left(m_{i}, m_{j}\right)$, the cosine value of evidence angle $\cos \left(m_{i}, m_{j}\right)$, and the similarity measure $\operatorname{sim}\left(m_{i}, m_{j}\right)$ between any two BOEs $m_{i}, m_{j}(i, j=1,2,3)$. After having 
TABLE 5: The evidence distance measure between BOEs.

\begin{tabular}{lcccc}
\hline Item & $m_{1}, m_{2}$ & $m_{1}, m_{2}, m_{3}$ & $m_{1}, m_{2}, m_{3}, m_{4}$ & $m_{1}, m_{2}, m_{3}, m_{4}, m_{5}$ \\
\hline$d\left(m_{1}, m_{2}\right)$ & 0.5386 & 0.5386 & 0.5386 & 0.5386 \\
$d\left(m_{1}, m_{3}\right)$ & - & 0.3495 & 0.3495 & 0.3495 \\
$d\left(m_{1}, m_{4}\right)$ & - & - & 0.3257 & 0.3257 \\
$d\left(m_{1}, m_{5}\right)$ & - & - & - & 0.3142 \\
$d\left(m_{2}, m_{3}\right)$ & - & 0.8142 & 0.7850 & 0.8142 \\
$d\left(m_{2}, m_{4}\right)$ & - & - & - & 0.7850 \\
$d\left(m_{2}, m_{5}\right)$ & - & - & 0.0300 & 0.7906 \\
$d\left(m_{3}, m_{4}\right)$ & - & - & - & 0.0300 \\
$d\left(m_{3}, m_{5}\right)$ & - & - & - & 0.0374 \\
$d\left(m_{4}, m_{5}\right)$ & - & & & 0.0354 \\
\hline
\end{tabular}

TABLE 6: The cosine value of evidence angle measure between BOEs.

\begin{tabular}{lcccc}
\hline Item & $m_{1}, m_{2}$ & $m_{1}, m_{2}, m_{3}$ & $m_{1}, m_{2}, m_{3}, m_{4}$ & $m_{1}, m_{2}, m_{3}, m_{4}, m_{5}$ \\
\hline $\cos \left(m_{1}, m_{2}\right)$ & 0.4504 & 0.4504 & 0.4504 & 0.4504 \\
$\cos \left(m_{1}, m_{3}\right)$ & - & 0.8399 & 0.8399 & 0.8399 \\
$\cos \left(m_{1}, m_{4}\right)$ & - & - & 0.8604 & 0.8604 \\
$\cos \left(m_{1}, m_{5}\right)$ & - & - & - & 0.8454 \\
$\cos \left(m_{2}, m_{3}\right)$ & - & 0.1142 & 0.1142 & 0.1142 \\
$\cos \left(m_{2}, m_{4}\right)$ & - & - & - & 0.1578 \\
$\cos \left(m_{2}, m_{5}\right)$ & - & - & 0.9990 & 0.1503 \\
$\cos \left(m_{3}, m_{4}\right)$ & - & - & - & 0.9990 \\
$\cos \left(m_{3}, m_{5}\right)$ & - & - & - & 0.9987 \\
$\cos \left(m_{4}, m_{5}\right)$ & & & & 0.9992 \\
\hline
\end{tabular}

TABLE 7: The similarity measure between BOEs.

\begin{tabular}{lcccc}
\hline Item & $m_{1}, m_{2}$ & $m_{1}, m_{2}, m_{3}$ & $m_{1}, m_{2}, m_{3}, m_{4}$ & $m_{1}, m_{2}, m_{3}, m_{4}, m_{5}$ \\
\hline $\operatorname{sim}\left(m_{1}, m_{2}\right)$ & 0.2078 & 0.2078 & 0.2078 & 0.2078 \\
$\operatorname{sim}\left(m_{1}, m_{3}\right)$ & - & 0.5464 & 0.5464 & 0.5464 \\
$\operatorname{sim}\left(m_{1}, m_{4}\right)$ & - & - & 0.5802 & 0.5802 \\
$\operatorname{sim}\left(m_{1}, m_{5}\right)$ & - & - & - & 0.0212 \\
$\operatorname{sim}\left(m_{2}, m_{3}\right)$ & - & 0.0212 & 0.0339 & 0.0212 \\
$\operatorname{sim}\left(m_{2}, m_{4}\right)$ & - & - & - & 0.0339 \\
$\operatorname{sim}\left(m_{2}, m_{5}\right)$ & - & - & 0.9690 & 0.0315 \\
$\operatorname{sim}\left(m_{3}, m_{4}\right)$ & - & - & - & 0.9690 \\
$\operatorname{sim}\left(m_{3}, m_{5}\right)$ & - & - & - & 0.9613 \\
$\operatorname{sim}\left(m_{4}, m_{5}\right)$ & & & & 0.9638 \\
\hline
\end{tabular}

TABLE 8: The support degree of each BOE.

\begin{tabular}{lcccc}
\hline Item & $m_{1}, m_{2}$ & $m_{1}, m_{2}, m_{3}$ & $m_{1}, m_{2}, m_{3}, m_{4}$ & $m_{1}, m_{2}, m_{3}, m_{4}, m_{5}$ \\
\hline $\sup \left(m_{1}\right)$ & 0.2078 & 0.3771 & 0.4448 & 0.4750 \\
$\sup \left(m_{2}\right)$ & 0.2078 & 0.1145 & 0.0876 & 0.0736 \\
$\sup \left(m_{3}\right)$ & - & 0.2838 & 0.5122 & 0.6245 \\
$\sup \left(m_{4}\right)$ & - & - & 0.5277 & 0.6367 \\
$\sup \left(m_{5}\right)$ & - & - & - & 0.6305 \\
\hline
\end{tabular}

$\operatorname{sim}\left(m_{i}, m_{j}\right)$, the support degree $\sup \left(m_{i}\right)$ and the initial weight $i w\left(m_{i}\right)$ of one BOE $m_{i}(i=1,2,3)$ could be obtained. The following tables from Tables 18 to 21 show the results of indexes mentioned above.
Next, based on the improved entropy function, the uncertainty degree $u\left(m_{i}\right)$ and the normalized uncertainty measure $u n\left(m_{i}\right)$ of each BOE could be computed well, as shown in Table 22. Finally, make use of equations 
TABLE 9: The initial weight determined by the evidence angle and distance functions of each BOE.

\begin{tabular}{lcccc}
\hline Items & $m_{1}, m_{2}$ & $m_{1}, m_{2}, m_{3}$ & $m_{1}, m_{2}, m_{3}, m_{4}$ & $m_{1}, m_{2}, m_{3}, m_{4}, m_{5}$ \\
\hline $\operatorname{iw}\left(m_{1}\right)$ & 0.5000 & 0.4863 & 0.2829 & 0.1946 \\
$\operatorname{iw}\left(m_{2}\right)$ & 0.5000 & 0.1477 & 0.0557 & 0.0302 \\
$\operatorname{iw}\left(m_{3}\right)$ & - & 0.3660 & 0.3258 & 0.2559 \\
$\operatorname{iw}\left(m_{4}\right)$ & - & - & 0.3356 & 0.2609 \\
$\operatorname{iw}\left(m_{5}\right)$ & - & - & - & 0.2584 \\
\hline
\end{tabular}

TABLE 10: The uncertainty measure of each BOE.

\begin{tabular}{lcccc}
\hline Item & $m_{1}, m_{2}$ & $m_{1}, m_{2}, m_{3}$ & $m_{1}, m_{2}, m_{3}, m_{4}$ & $m_{1}, m_{2}, m_{3}, m_{4}, m_{5}$ \\
\hline$u\left(m_{1}\right)$ & 3.5317 & 3.5317 & 3.5317 & 3.5317 \\
$u\left(m_{2}\right)$ & 2.0540 & 2.0540 & 2.0540 & 2.0540 \\
$u\left(m_{3}\right)$ & - & 3.0442 & 3.0442 & 3.0442 \\
$u\left(m_{4}\right)$ & - & - & 3.1264 & 3.1264 \\
$u\left(m_{5}\right)$ & - & - & - & 3.0559 \\
\hline
\end{tabular}

TABLE 11: The normalization of uncertainty measure of each BOE.

\begin{tabular}{lcccc}
\hline Item & $m_{1}, m_{2}$ & $m_{1}, m_{2}, m_{3}$ & $m_{1}, m_{2}, m_{3}, m_{4}$ & $m_{1}, m_{2}, m_{3}, m_{4}, m_{5}$ \\
\hline $\operatorname{un}\left(m_{1}\right)$ & 0.6323 & 0.4092 & 0.3004 & 0.2384 \\
$\operatorname{un}\left(m_{2}\right)$ & 0.3677 & 0.2380 & 0.1747 & 0.1387 \\
$\operatorname{un}\left(m_{3}\right)$ & - & 0.3528 & 0.2590 & 0.2055 \\
$\operatorname{un}\left(m_{4}\right)$ & - & - & 0.2659 & 0.2111 \\
$\operatorname{un}\left(m_{5}\right)$ & - & - & - & 0.2063 \\
\hline
\end{tabular}

TABLE 12: The final weight determined by the improved entropy function.

\begin{tabular}{lcccc}
\hline Item & $m_{1}, m_{2}$ & $m_{1}, m_{2}, m_{3}$ & $m_{1}, m_{2}, m_{3}, m_{4}$ & $m_{1}, m_{2}, m_{3}, m_{4}, m_{5}$ \\
\hline $\mathrm{fw}\left(m_{1}\right)$ & 0.4342 & 0.4637 & 0.2739 & 0.1894 \\
$\mathrm{fw}\left(m_{2}\right)$ & 0.5658 & 0.1671 & 0.0611 & 0.0325 \\
$\mathrm{fw}\left(m_{3}\right)$ & - & 0.3692 & 0.3287 & 0.2574 \\
$\mathrm{fw}\left(m_{4}\right)$ & - & - & 0.3363 & 0.2610 \\
$\mathrm{fw}\left(m_{5}\right)$ & - & - & - & 0.2597 \\
\hline
\end{tabular}

TABLE 13: The results of WAM (m).

\begin{tabular}{lcccc}
\hline Item & $m_{1}, m_{2}$ & $m_{1}, m_{2}, m_{3}$ & $m_{1}, m_{2}, m_{3}, m_{4}$ & $m_{1}, m_{2}, m_{3}, m_{4}, m_{5}$ \\
\hline & $m\left(\theta_{1}\right)=0.1780$ & $m\left(\theta_{1}\right)=0.4043$ & $m\left(\theta_{1}\right)=0.4879$ & $m\left(\theta_{1}\right)=0.5263$ \\
WAM $(\mathrm{m})$ & $m\left(\theta_{2}\right)=0.6351$ & $m\left(\theta_{2}\right)=0.3107$ & $m\left(\theta_{2}\right)=0.1911$ & $m\left(\theta_{2}\right)=0.1543$ \\
& $m\left(\theta_{3}\right)=0.1869$ & $m\left(\theta_{3}\right)=0.1558$ & $m\left(\theta_{3}\right)=0.0883$ & $m\left(\theta_{3}\right)=0.0600$ \\
& $m\left(\theta_{1}, \theta_{3}\right)=0$ & $m\left(\theta_{1}, \theta_{3}\right)=0.1292$ & $m\left(\theta_{1}, \theta_{3}\right)=0.2327$ & $m\left(\theta_{1}, \theta_{3}\right)=0.2594$ \\
\hline
\end{tabular}

TABLE 14: The final final BPAs calculated by the newly proposed approach.

\begin{tabular}{lcccc}
\hline Item & $m_{1}, m_{2}$ & $m_{1}, m_{2}, m_{3}$ & $m_{1}, m_{2}, m_{3}, m_{4}$ & $m_{1}, m_{2}, m_{3}, m_{4}, m_{5}$ \\
\hline & $m\left(\theta_{1}\right)=0.0674$ & $m\left(\theta_{1}\right)=0.7380$ & $m\left(\theta_{1}\right)=0.9571$ & $m\left(\theta_{1}\right)=0.9987$ \\
Final BPA & $m\left(\theta_{2}\right)=0.8583$ & $m\left(\theta_{2}\right)=0.1479$ & $m\left(\theta_{2}\right)=0.0048$ & $m\left(\theta_{2}\right)=0.0001$ \\
& $m\left(\theta_{3}\right)=0.0743$ & $m\left(\theta_{3}\right)=0.1035$ & $m\left(\theta_{3}\right)=0.0276$ & $m\left(\theta_{3}\right)=0.0007$ \\
& $m\left(\theta_{1}, \theta_{3}\right)=0$ & $m\left(\theta_{1}, \theta_{3}\right)=0.0106$ & $m\left(\theta_{1}, \theta_{3}\right)=0.0105$ & $m\left(\theta_{1}, \theta_{3}\right)=0.0005$ \\
\hline
\end{tabular}


TABle 15: The comparison between our newly proposed method and other current mainly popular methods.

\begin{tabular}{|c|c|c|c|c|}
\hline \multirow{2}{*}{ Approach } & \multicolumn{4}{|c|}{ Combination outcomes } \\
\hline & $m_{1}, m_{2}$ & $m_{1}, m_{2}, m_{3}$ & $m_{1}, m_{2}, m_{3}, m_{4}$ & $m_{1}, m_{2}, m_{3}, m_{4}, m_{5}$ \\
\hline Dempster's rule [1] & $\begin{array}{c}m\left(\theta_{1}\right)=0 \\
m\left(\theta_{2}\right)=0.8969 \\
m\left(\theta_{3}\right)=0.1031\end{array}$ & $\begin{array}{c}m\left(\theta_{1}\right)=0 \\
m\left(\theta_{2}\right)=0.6575 \\
m\left(\theta_{3}\right)=0.3425\end{array}$ & $\begin{array}{c}m\left(\theta_{1}\right)=0 \\
m\left(\theta_{2}\right)=0.3321 \\
m\left(\theta_{3}\right)=0.6679\end{array}$ & $\begin{array}{c}m\left(\theta_{1}\right)=0 \\
m\left(\theta_{2}\right)=0.1422 \\
m\left(\theta_{3}\right)=0.8575\end{array}$ \\
\hline Murphy's simple average [33] & $\begin{array}{c}m\left(\theta_{1}\right)=0.0964 \\
m\left(\theta_{2}\right)=0.8119 \\
m\left(\theta_{3}\right)=0.0917 \\
m\left(\theta_{1}, \theta_{3}\right)=0\end{array}$ & $\begin{array}{c}m\left(\theta_{1}\right)=0.4619 \\
m\left(\theta_{2}\right)=0.4497 \\
m\left(\theta_{3}\right)=0.0794 \\
m\left(\theta_{1}, \theta_{3}\right)=0.0090\end{array}$ & $\begin{array}{c}m\left(\theta_{1}\right)=0.8362 \\
m\left(\theta_{2}\right)=0.1147 \\
m\left(\theta_{3}\right)=0.0410 \\
m\left(\theta_{1}, \theta_{3}\right)=0.0081\end{array}$ & $\begin{array}{c}m\left(\theta_{1}\right)=0.9620 \\
m\left(\theta_{2}\right)=0.0210 \\
m\left(\theta_{3}\right)=0.0138 \\
m\left(\theta_{1}, \theta_{3}\right)=0.0032\end{array}$ \\
\hline Deng's weighted average [34] & $\begin{array}{c}m\left(\theta_{1}\right)=0.0964 \\
m\left(\theta_{2}\right)=0.8119 \\
m\left(\theta_{3}\right)=0.0917 \\
m\left(\theta_{1}, \theta_{3}\right)=0\end{array}$ & $\begin{array}{c}m\left(\theta_{1}\right)=0.4974 \\
m\left(\theta_{2}\right)=0.4054 \\
m\left(\theta_{3}\right)=0.0888 \\
m\left(\theta_{1}, \theta_{3}\right)=0.0084\end{array}$ & $\begin{array}{c}m\left(\theta_{1}\right)=0.9089 \\
m\left(\theta_{2}\right)=0.0444 \\
m\left(\theta_{3}\right)=0.0379 \\
m\left(\theta_{1}, \theta_{3}\right)=0.0089\end{array}$ & $\begin{array}{c}m\left(\theta_{1}\right)=0.9820 \\
m\left(\theta_{2}\right)=0.0039 \\
m\left(\theta_{3}\right)=0.0107 \\
m\left(\theta_{1}, \theta_{3}\right)=0.0034\end{array}$ \\
\hline Our last work [36] & $\begin{array}{c}m\left(\theta_{1}\right)=0.0846 \\
m\left(\theta_{2}\right)=0.8305 \\
m\left(\theta_{3}\right)=0.0849 \\
m\left(\theta_{1}, \theta_{3}\right)=0\end{array}$ & $\begin{array}{c}m\left(\theta_{1}\right)=0.5784 \\
m\left(\theta_{2}\right)=0.0253 \\
m\left(\theta_{3}\right)=0.0958 \\
m\left(\theta_{1}, \theta_{3}\right)=0.0081\end{array}$ & $\begin{array}{c}m\left(\theta_{1}\right)=0.9303 \\
m\left(\theta_{2}\right)=0.0129 \\
m\left(\theta_{3}\right)=0.0351 \\
m\left(\theta_{1}, \theta_{3}\right)=0.0093\end{array}$ & $\begin{array}{c}m\left(\theta_{1}\right)=0.9939 \\
m\left(\theta_{2}\right)=0.0005 \\
m\left(\theta_{3}\right)=0.0044 \\
m\left(\theta_{1}, \theta_{3}\right)=0.0012\end{array}$ \\
\hline Our newly proposed method & $\begin{array}{c}m\left(\theta_{1}\right)=0.0674 \\
m\left(\theta_{2}\right)=0.8583 \\
m\left(\theta_{3}\right)=0.0743 \\
m\left(\theta_{1}, \theta_{3}\right)=0\end{array}$ & $\begin{array}{c}m\left(\theta_{1}\right)=0.7380 \\
m\left(\theta_{2}\right)=0.1479 \\
m\left(\theta_{3}\right)=0.1035 \\
m\left(\theta_{1}, \theta_{3}\right)=0.0106\end{array}$ & $\begin{array}{c}m\left(\theta_{1}\right)=0.9571 \\
m\left(\theta_{2}\right)=0.0048 \\
m\left(\theta_{3}\right)=0.0276 \\
m\left(\theta_{1}, \theta_{3}\right)=0.0105\end{array}$ & $\begin{aligned} m\left(\theta_{1}\right) & =0.9987 \\
m\left(\theta_{2}\right) & =0.0001 \\
m\left(\theta_{3}\right) & =0.0007 \\
m\left(\theta_{1}, \theta_{3}\right) & =0.0005\end{aligned}$ \\
\hline
\end{tabular}

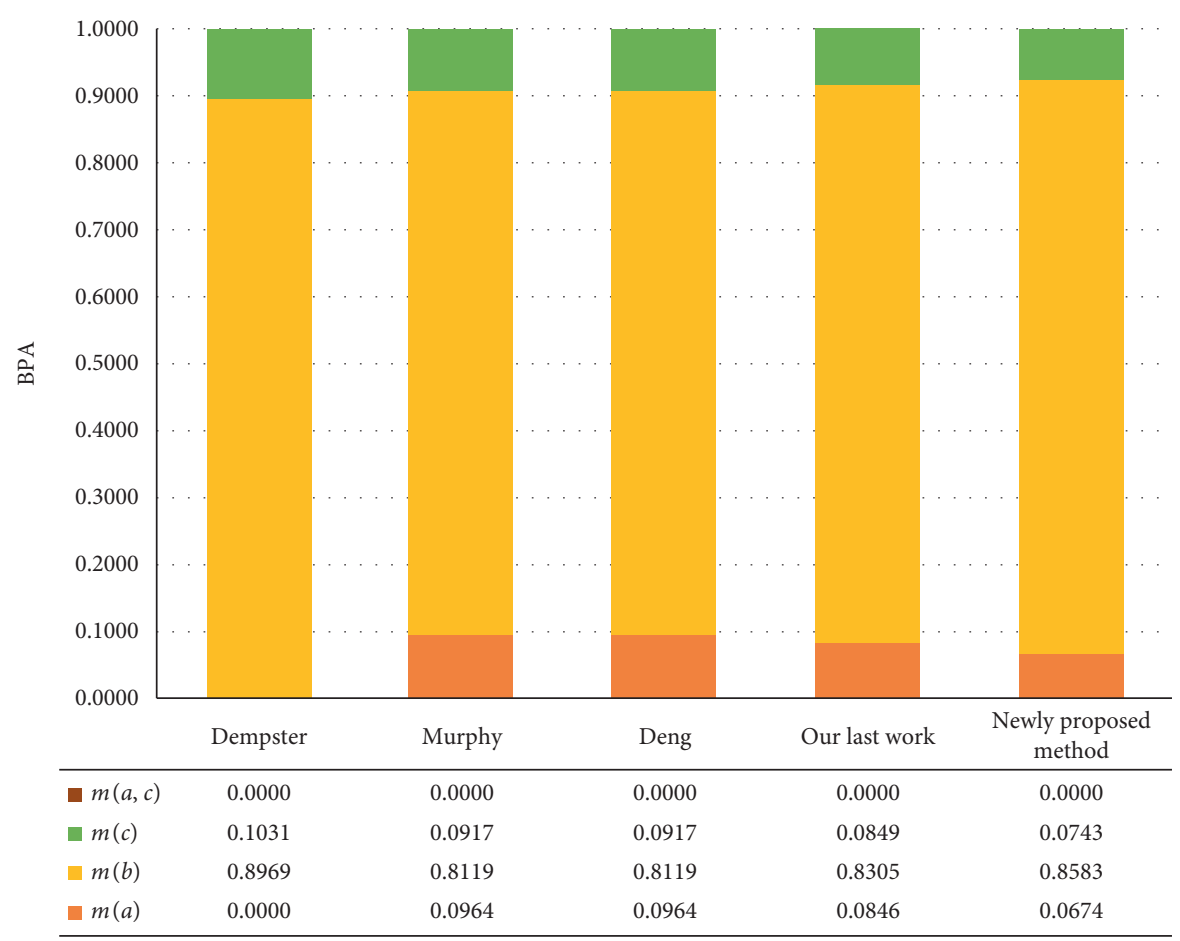

Figure 1: The combination results using different combination rules under condition of two BOEs.

(29) and (30) to modify the initial weight $i w\left(m_{i}\right)$ and get the final weight $f w\left(m_{i}\right)(i=1,2,3)$, i.e., the dynamic reliability $R_{i}^{d}$. The results of $R_{i}^{d}$ are listed in Table 23.

So far, we have had the static reliability $R_{i}^{s}(i=1,2,3)$ and the dynamic reliability $R_{i}^{d}(i=1,2,3)$ of each BOE. The last step is to combine these two reliability indexes to get the final comprehensive sensor reliability
$R_{i}(i=1,2,3)$ on the basis of the formula $R_{i}=R_{i}^{s} \times R_{i}^{d}$ and normalize it, denoted as $R N_{i}$. The results are shown in Table 24.

At last, modify BPAs with comprehensive sensor reliability $R N$ to get WAM $(m)$, as shown in Table 25 , and then, using the classical combination rule to combine WAM $(m)$ for two times, we will get final results listed in Table 26. 


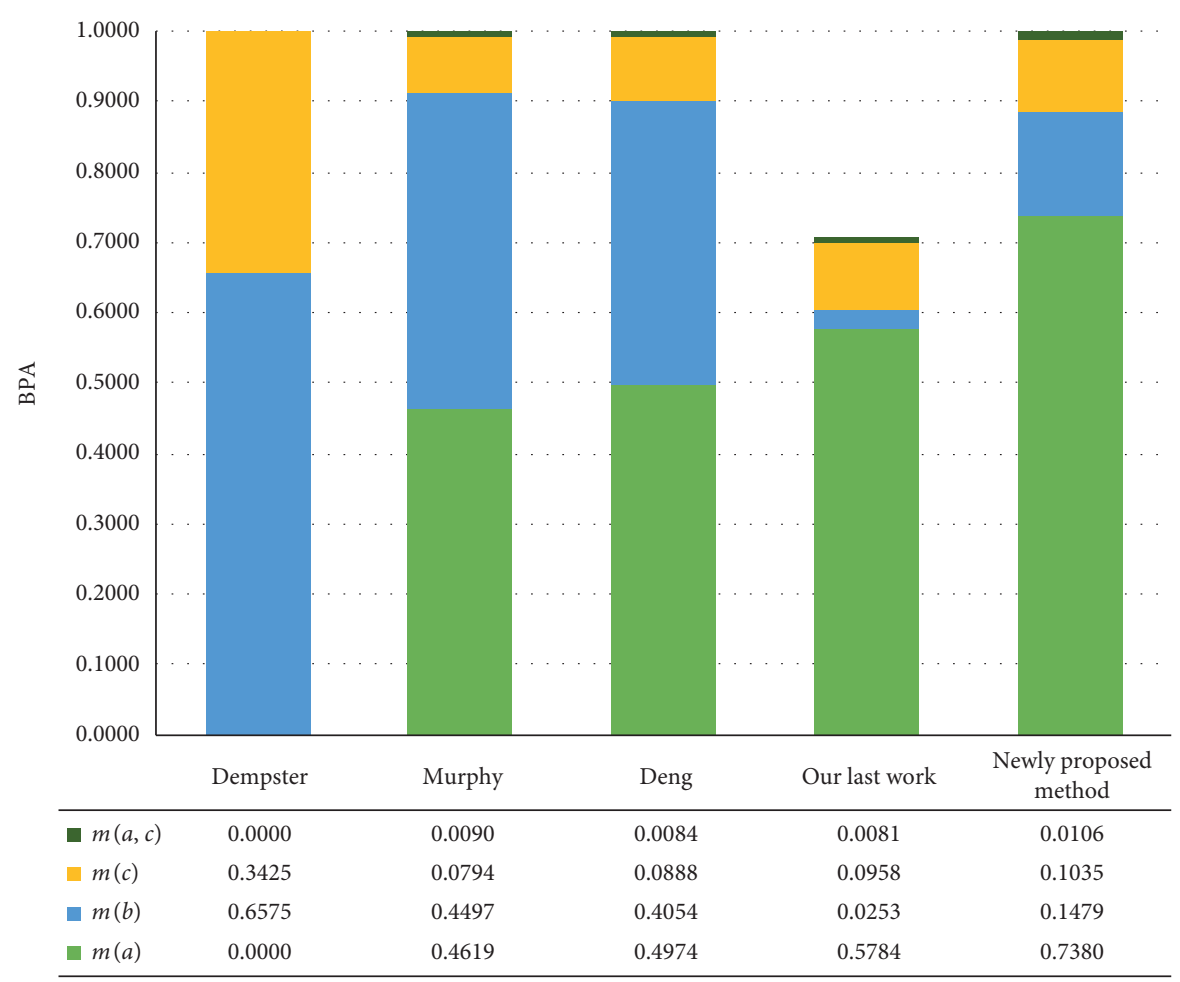

FIgURE 2: The combination results using different combination rules under condition of three BOEs.

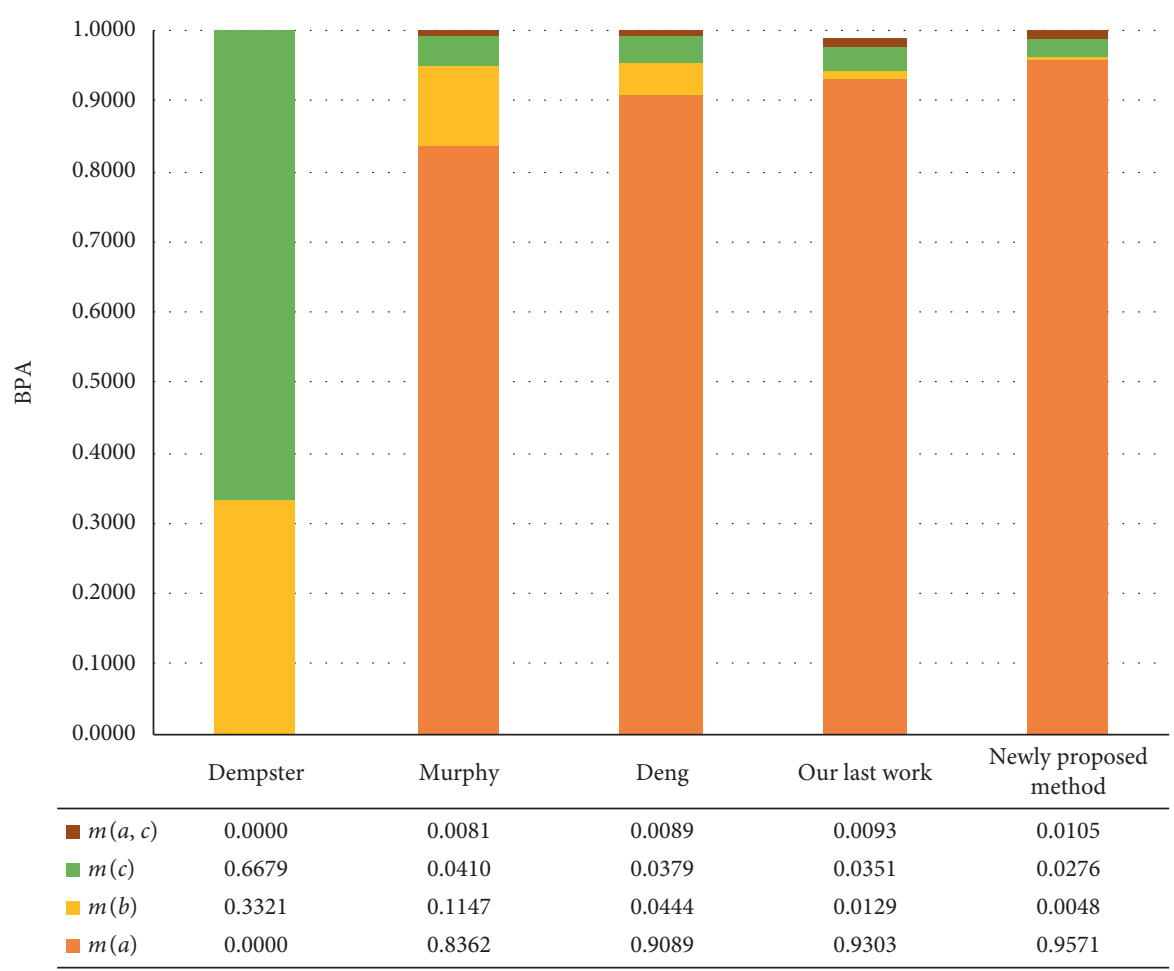

FIGURE 3: The combination results using different combination rules under condition of four BOEs.

As shown in Table 26, our newly proposed method assigns the fault mode $F_{1} 95.42 \%$ of total belief, and the fault mode $F_{2}$ only owns $4.84 \%$ belief. What we also concern is that the uncertainty of belief $m(\Omega)$ has been reduced to 0.0012 . In a word, this latest approach can provide a pretty precise combined result for decision-makers. 


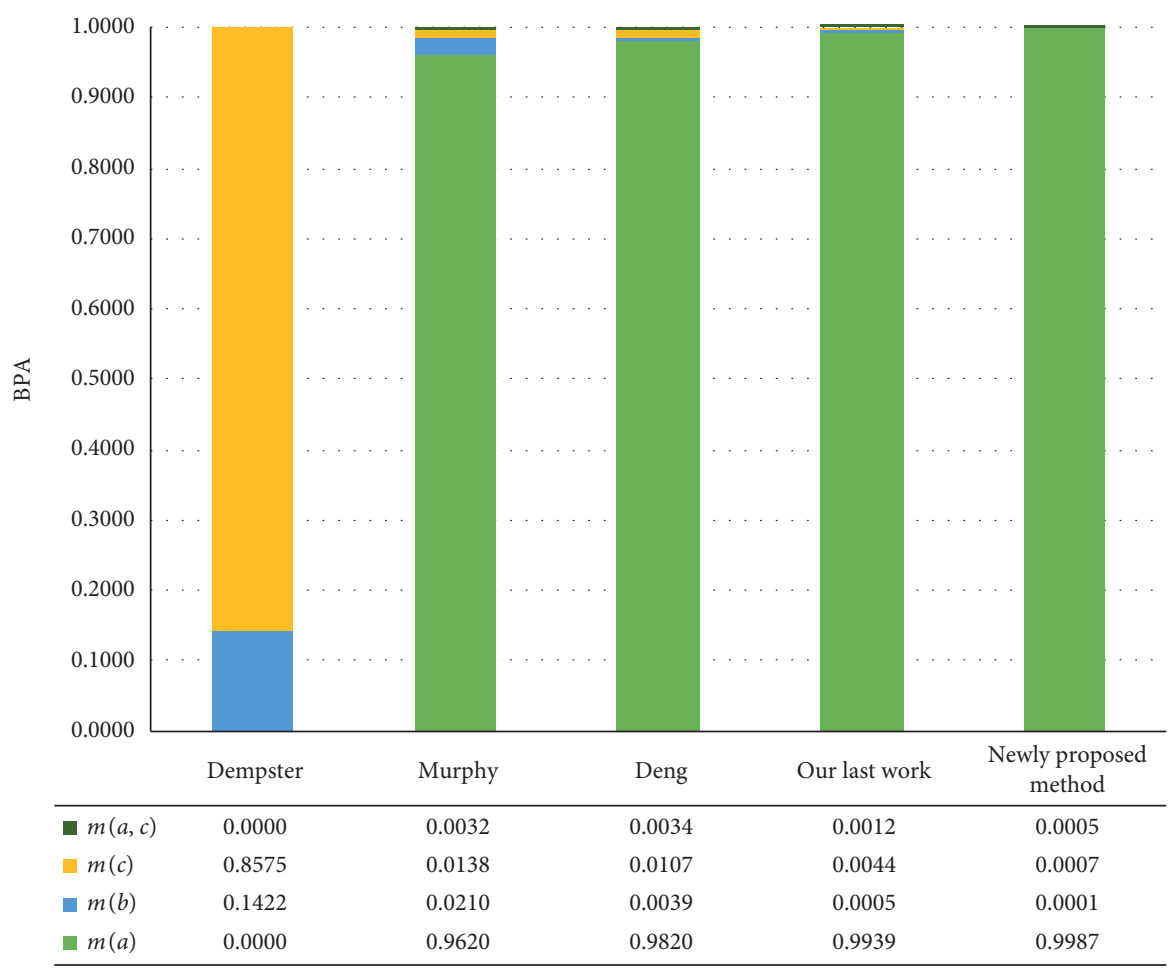

FIGURE 4: The combination results using different combination rules under condition of five BOEs.

TABLE 16: The prior information of collected BOEs.

\begin{tabular}{|c|c|c|c|c|c|c|}
\hline Evidences & $m\left(F_{1}\right)$ & $m\left(F_{2}\right)$ & $m\left(F_{2}, F_{3}\right)$ & $m(\Omega)$ & $\operatorname{suf}(\mu)$ & $\operatorname{imp}(\nu)$ \\
\hline$m_{1}$ & 0.6 & 0.1 & 0.1 & 0.2 & 1 & 1 \\
\hline$m_{2}$ & 0.05 & 0.8 & 0.05 & 0.1 & 0.6 & 0.34 \\
\hline$m_{3}$ & 0.7 & 0.1 & 0.1 & 0.1 & 1 & 1 \\
\hline
\end{tabular}

TABLE 17: The static reliability of BOEs in fault diagnosis application.

\begin{tabular}{cccc}
\hline & $m_{1}$ & $m_{2}$ & $m_{3}$ \\
\hline$R_{i}^{s}$ & 1 & 0.204 & 1 \\
\hline
\end{tabular}

TABLE 18: The evidence distance measure between BOEs in fault diagnosis application.

\begin{tabular}{lccc}
\hline Item & $d\left(m_{1}, m_{2}\right)$ & $d\left(m_{1}, m_{3}\right)$ & $d\left(m_{2}, m_{3}\right)$ \\
\hline Value & 0.6185 & 0.0817 & 0.6633 \\
\hline
\end{tabular}

This newly proposed method is also compared with others including two of our previous works and the related comparison results are shown in Table 27 and Figure 5. As seen from Table 27 and Figure 5, we easily find the latest method has significantly more belief degree of fault mode $F_{1}$ and less uncertainty of belief than those of Fan and Zuo [70] and our previous works [35, 36]. That is because these three efficient tools, evidence distance, evidence angle, and improved entropy, compute dynamic reliability of each sensor more reasonably and comprehensively and reduce the conflicts among BOEs to the maximum extent. 
TABLE 19: The cosine value of the evidence angle measure between BOEs in fault diagnosis application.

\begin{tabular}{lccc}
\hline Item & $\cos \left(m_{1}, m_{2}\right)$ & $\cos \left(m_{1}, m_{3}\right)$ & $\cos \left(m_{2}, m_{3}\right)$ \\
\hline Value & 0.4043 & 0.9961 & 0.3398 \\
\hline
\end{tabular}

TABLE 20: The similarity measure between BOEs in fault diagnosis application.

\begin{tabular}{lccc}
\hline Item & $\operatorname{sim}\left(m_{1}, m_{2}\right)$ & $\operatorname{sim}\left(m_{1}, m_{3}\right)$ & $\operatorname{sim}\left(m_{2}, m_{3}\right)$ \\
\hline Value & 0.1543 & 0.9148 & 0.1144 \\
\hline
\end{tabular}

TABLE 21: The support degree and initial weight of each BOE in fault diagnosis application.

\begin{tabular}{lccc}
\hline BOE & $m_{1}$ & $m_{2}$ & $m_{3}$ \\
\hline $\sup \left(m_{i}\right)$ & 0.5345 & 0.1343 & 0.5146 \\
$\operatorname{iw}\left(m_{i}\right)$ & 0.4517 & 0.1135 & 0.4348 \\
\hline
\end{tabular}

TABLE 22: The uncertainty measure of each BOE in fault diagnosis application.

\begin{tabular}{lccr}
\hline BOE & $m_{1}$ & $m_{2}$ & $m_{3}$ \\
\hline$u\left(m_{i}\right)$ & 3.4589 & 2.7584 & 3.1225 \\
un $\left(m_{i}\right)$ & 0.3704 & 0.2953 & 0.3343 \\
\hline
\end{tabular}

TABLe 23: The dynamic reliability of BOEs in fault diagnosis application.

\begin{tabular}{lccc}
\hline BOE & $m_{1}$ & $m_{2}$ & $m_{3}$ \\
\hline$f w\left(m_{i}\right)$ & 0.4408 & 0.1194 & 0.4398 \\
$R_{i}^{d}$ & 0.4408 & 0.1194 & 0.4398 \\
\hline
\end{tabular}

TABLE 24: The final comprehensive sensor reliability in fault diagnosis application.

\begin{tabular}{lccr}
\hline BOE & $m_{1}$ & $m_{2}$ & $m_{3}$ \\
\hline$R_{i}$ & 0.4408 & 0.0244 & 0.4398 \\
$R N_{i}$ & 0.4871 & 0.0269 & 0.4860 \\
\hline
\end{tabular}

TABLE 25: The $\operatorname{WAM}(m)$ results in fault diagnosis application.

\begin{tabular}{llccc}
\hline & $m\left(F_{1}\right)$ & $m\left(F_{2}\right)$ & $m\left(F_{2}, F_{3}\right)$ & $m(\Omega)$ \\
\hline $\mathrm{WAM}(\mathrm{m})$ & 0.6338 & 0.1188 & 0.0987 & 0.1487 \\
\hline
\end{tabular}

TABLe 26: The final final results in fault diagnosis application.

\begin{tabular}{lcccc}
\hline & $m\left(F_{1}\right)$ & $m\left(F_{2}\right)$ & $m\left(F_{2}, F_{3}\right)$ & $m(\Omega)$ \\
\hline VALUE & 0.9542 & 0.0363 & 0.0083 & 0.0012 \\
\hline
\end{tabular}

TABLE 27: The comparison results in application.

\begin{tabular}{lccc}
\hline & $m\left(F_{1}\right)$ & $m\left(F_{2}\right)$ & $m\left(F_{2}, F_{3}\right)$ \\
\hline Classical D-S theory [1] & 0.4519 & 0.5048 & 0.0336 \\
Fan and Zuo [70] & 0.8119 & 0.1096 & 0.0526 \\
Our previous work no.1 [35] & 0.8899 & 0.0785 & 0.0243 \\
Our previous work no.2 [36] & 0.9416 & 0.0484 & 0.0087 \\
The newly proposed method & 0.9542 & 0.0363 & 0.0259 \\
\hline
\end{tabular}




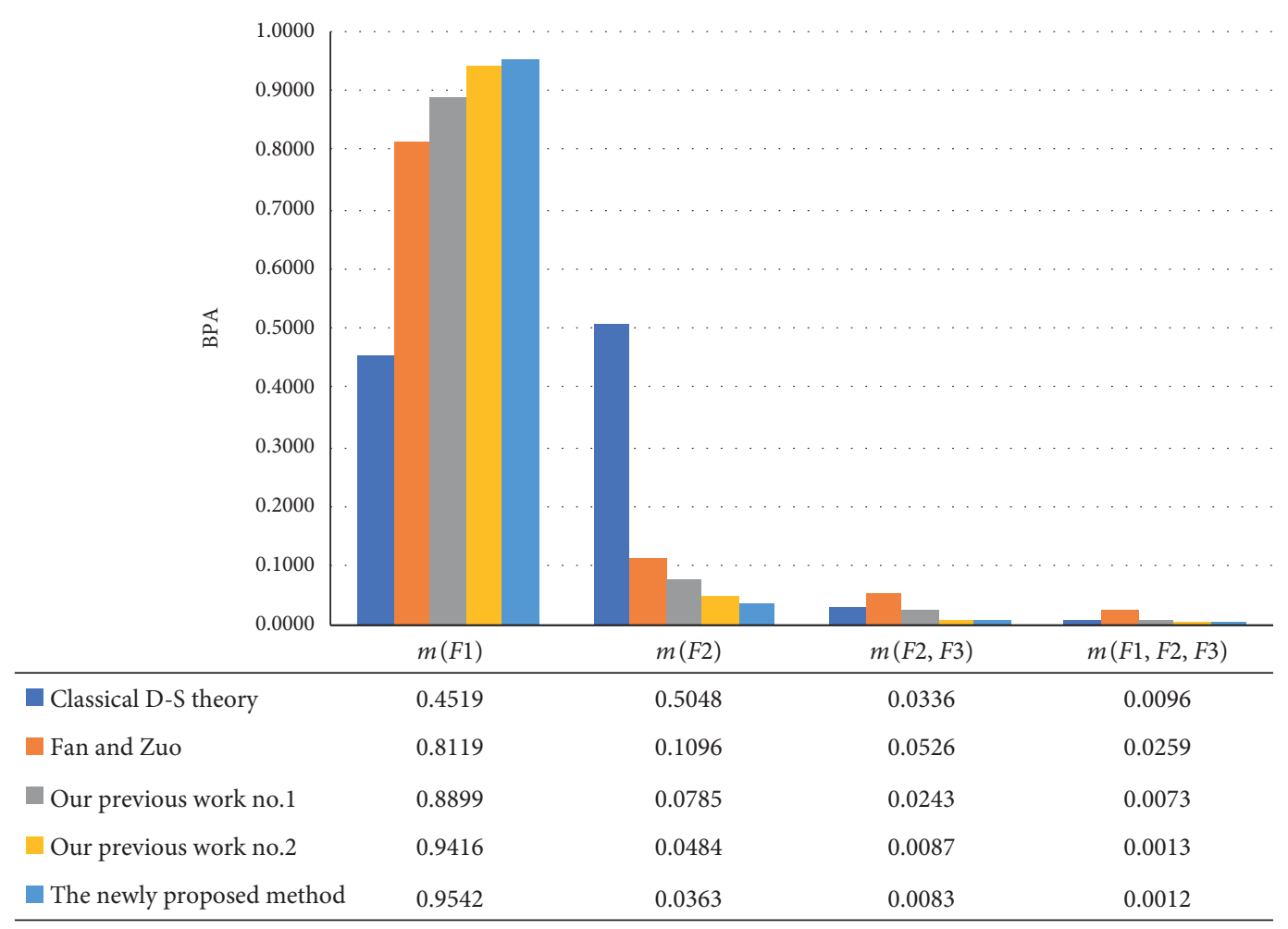

Figure 5: The comparison results of different combination rules in fault diagnosis application.

\section{Conclusion}

In this paper, a new method to handle conflict when combining evidences is presented. Compared to our previous works $[35,36]$, the evidence angle is added to this new work in order to describe the consistency degree between the evidences. This newly proposed approach consists of three steps: firstly, both the evidence distance and evidence angle determine the initial weight of each BOE; secondly, the improved entropy is used for modifying the initial weight; finally, apply the classical D-S combination rule to get final fusion results. Moreover, one numeric example and one fault diagnosis application sufficiently demonstrate the efficiency and effectiveness of this new method, and the related comparison results show our proposed approach can converge fast and reduce most uncertainty of decision-making when handling highly conflicting evidences, and so it can help experts make a better and faster decision.

\section{Data Availability}

All the data used in this study are available within the manuscript.

\section{Conflicts of Interest}

The authors declare that there are no conflicts of interest regarding the publication of this paper.

\section{Acknowledgments}

This work was supported by Specialized Research Fund for the Doctoral Program of Higher Education of China (SRFDP) (no.
20130191110027), by Research and Construction of Self-Regulated Learning Platform of Software Technology Specialty in Higher Vocational Colleges (no. KJ1 502901), and by Vulnerability Analysis and Security Audit Reinforcement Based on Docker Container (no. 2018A01008).

\section{References}

[1] A. P. Dempster, "Upper and lower probabilities induced by a multivalued mapping," The Annals of Mathematical Statistics, vol. 38, no. 2, pp. 325-339, 1967.

[2] G. Shafer, A Mathematical Theory of Evidence, Princeton University Press, Princeton, UK, 1976.

[3] W. Jiang, C. Xie, M. Zhuang, and Y. Tang, "Failure mode and effects analysis based on a novel fuzzy evidential method," Applied Soft Computing, vol. 57, pp. 672-683, 2017.

[4] X. Deng, W. Jiang, and Z. Wang, "Zero-sum polymatrix games with link uncertainty: a dempster-shafer theory solution," Applied Mathematics and Computation, vol. 340, pp. 101-112, 2019.

[5] F. Xiao, "A new divergence measure for belief functions in D-S evidence theory for multisensor data fusion," Information Sciences, vol. 514, pp. 462-483, 2020.

[6] Y. Tang, D. Zhou, S. Xu, and Z. He, "A weighted belief entropy-based uncertainty measure for multi-sensor data fusion," Sensors, vol. 17, no. 4, p. 928, 2017.

[7] T. Denoeux, "Logistic regression, neural networks and dempster-shafer theory: a new perspective," Knowledge-Based Systems, vol. 176, pp. 54-67, 2019.

[8] Y. Zhou, Y. Tang, and X. Zhao, "A novel uncertainty management approach for air combat situation assessment based on improved belief entropy," Entropy, vol. 21, no. 5, p. 495, 2019. 
[9] F. Xiao, "Multi-sensor data fusion based on the belief divergence measure of evidences and the belief entropy," Information Fusion, vol. 46, pp. 23-32, 2019.

[10] G. Shafer, A Mathematical Theory of Evidence, Princeton University Press, Princeton, UK, 1976.

[11] D. Suh and J. Yook, "A method to determine basic probability assignment in context awareness of a moving object," International Journal of Distributed Sensor Networks, vol. 9, no. 8, p. 972641, 2013.

[12] W. Zhu, H. Yang, Y. Jin, and B. Liu, "A method for recognizing fatigue ddriving based on ddempster-shafer theory and fuzzy neural network," Mathematical Problems in Engineering, vol. 9, 2017.

[13] H. Zheng and Y. Deng, "Evaluation method based on fuzzy relations between Dempster-Shafer belief structure," International Journal of Intelligent Systems, vol. 33, no. 7, pp. 1343-1363, 2018.

[14] F. Liu and Y. Deng, "Determine the number of unknown targets in open world based on elbow method," IEEE Transactions on Fuzzy Systems, vol. 33, 2018.

[15] H.-C. Liu, J.-X. You, X.-J. Fan, and Q.-L. Lin, "Failure mode and effects analysis using $\mathrm{D}$ numbers and grey relational projection method," Expert Systems with Applications, vol. 41, no. 10, pp. 4670-4679, 2014.

[16] X. Su, S. Mahadevan, P. Xu, and Y. Deng, "Dependence assessment in Human Reliability Analysis using evidence theory and AHP," Risk Analysis, vol. 35, no. 7, pp. 1296-1316, 2015.

[17] H. Zhang and Y. Deng, "Weighted belief function of sensor data fusion in engine fault diagnosis," Soft Computing, vol. 24, no. 3, pp. 2329-2339, 2020.

[18] L. Fei, Y. Deng, and Y. Hu, "Ds-vikor: a new multi-criteria decision-making method for supplier selection," International Journal of Fuzzy Systems, vol. 21, no. 1, pp. 157-175, 2019.

[19] R. Li, H. Li, and Y. Tang, "An improved method to manage conflict data using elementary belief assignment function in the evidence theory," IEEE Access, vol. 8, pp. 37926-37932, 2020.

[20] W. Jiang, Y. Yang, Y. Luo, and X. Qin, "Determining basic probability assignment based on the improved similarity measures of generalized fuzzy numbers," International Journal of Computers Communications \& Control, vol. 10, no. 3, pp. 333-347, 2015.

[21] J. Ma, W. Liu, P. Miller, and H. Zhou, "An evidential fusion approach for gender profiling," Information Sciences, vol. 333, pp. 10-20, 2016.

[22] X. Deng, Y. Hu, Y. Deng, and S. Mahadevan, "Supplier selection using AHP methodology extended by D numbers," Expert Systems with Applications, vol. 41, no. 1, pp. 156-167, 2014.

[23] L. Fei, J. Xia, Y. Feng, and L. Liu, "An electre-based multiple criteria decision making method for supplier selection using dempster-shafer theory," IEEE Access, vol. 7, pp. 84701-84716, 2019.

[24] Y. Deng, Y. Liu, and D. Zhou, "An improved genetic algorithm with initial population strategy for symmetric TSP," Mathematical Problems in Engineering, vol. 2015, p. 212794 , 2015.

[25] W.-B. Du, Y. Gao, C. Liu, Z. Zheng, and Z. Wang, “Adequate is better: particle swarm optimization with limited-information," Applied Mathematics and Computation, vol. 268, pp. 832-838, 2015.

[26] X. Deng and W. Jiang, "On the negation of a Dempster-Shafer belief structure based on maximum uncertainty allocation," Information Sciences, vol. 516, pp. 346-352, 2020.
[27] L. A. Zadeh, "A simple view of the dempster-shafer theory of evidence and its implication for the rule of combination," $A I$ Magazine, vol. 7, no. 2, p. 85, 1986.

[28] Y. Deng, "Generalized evidence theory," Applied Intelligence, vol. 43, no. 3, pp. 530-543, 2015.

[29] W. Zhang and Y. Deng, "Combining conflicting evidence using the dematel method," Soft Computing, vol. 43, pp. 1-10, 2018.

[30] F. Xiao and B. Qin, "A weighted combination method for conflicting evidence in multi-sensor data fusion," Sensors (Basel, Switzerland), vol. 18, no. 5, 2018.

[31] R. R. Yager, "On the dempster-shafer framework and new combination rules," Information Sciences, vol. 41, no. 2, pp. 93-137, 1987.

[32] K. Yamada, "A new combination of evidence based on compromise," Fuzzy Sets and Systems, vol. 159, no. 13, pp. 1689-1708, 2008.

[33] C. K. Murphy, "Combining belief functions when evidence conflicts," Decision Support Systems, vol. 29, no. 1, pp. 1-9, 2000.

[34] Y. Deng, W. Shi, Z. Zhu, and Q. Liu, "Combining belief functions based on distance of evidence," Decision Support Systems, vol. 38, no. 3, pp. 489-493, 2004.

[35] L. Chen, L. Diao, and J. Sang, "Weighted evidence combination rule based on evidence ddistance and uncertainty measure: an application in fault ddiagnosis," Mathematical Problems in Engineering, vol. 38, 2018.

[36] L. Chen, L. Diao, and J. Sang, "A novel weighted evidence combination rule based on improved entropy function with a diagnosis application," International Journal of Distributed Sensor Networks, vol. 15, no. 1, p. 1550147718823990, 2019.

[37] P. Smets, "Analyzing the combination of conflicting belief functions," Information Fusion, vol. 8, no. 4, pp. 387-412, 2007.

[38] J. Wang, F. Xiao, X. Deng, L. Fei, and Y. Deng, "Weighted evidence combination based on distance of evidence and entropy function," International Journal of Ddistributed Sensor Networks, vol. 12, no. 7, p. 3218784, 2016.

[39] F. Xiao, "Efmcdm: evidential fuzzy multicriteria decision making based on belief entropy," IEEE Transactions on Fuzzy Systems, vol. 38, 2020.

[40] S. Li, F. Xiao, and J. H. Abawajy, "Conflict management of evidence theory based on belief entropy and negation," IEEE Access, vol. 8, pp. 37766-37774, 2020.

[41] P. Smets, "The combination of evidence in the transferable belief model," IEEE Transactions on Pattern Analysis and Machine Intelligence, vol. 12, no. 5, pp. 447-458, 1990.

[42] D. Dubois and H. Prade, "Representation and combination of uncertainty with belief functions and possibility measures," Computational Intelligence, vol. 4, no. 3, pp. 244-264, 1988.

[43] D. Han, Y. Deng, C. Han, and Z. Hou, "Weighted evidence combination based on distance of evidence and uncertainty measure," Journal of Infrared, Millimeter, and Terahertz Waves, vol. 30, pp. 396-400, 2011.

[44] A.-L. Jousselme and P. Maupin, On Some Properties of Ddistances in Evidence Theory, Belief, Brest, France, 2016.

[45] Y. Yang and D. Han, "A new distance-based total uncertainty measure in the theory of belief functions," Knowledge-Based Systems, vol. 94, pp. 114-123, 2016.

[46] D. Fixsen and R. P. S. Mahler, "The modified Dempster-Shafer approach to classification," IEEE Transactions on Systems, Man, and Cybernetics - Part A: Systems and Humans, vol. 27, no. 1, pp. 96-104, 1997. 
[47] H. Guo, W. Shi, and Y. Deng, "Evaluating sensor reliability in classification problems based on evidence theory," IEEE Transactions on Systems, Man and Cybernetics, Part B (Cybernetics), vol. 36, no. 5, pp. 970-981, 2006.

[48] B. Ristic and P. Smets, "The tbm global distance measure for the association of uncertain combat id declarations," Information Fusion, vol. 7, no. 3, pp. 276-284, 2006.

[49] A.-L. Jousselme, D. Grenier, and É. Bossé, "A new distance between two bodies of evidence," Information Fusion, vol. 2, no. 2, pp. 91-101, 2001.

[50] C. Wen, Y. Wang, and X. Xu, "Fuzzy information fusion algorithm of fault diagnosis based on similarity measure of evidence," in Advances in Neural Networks-ISNN 2008, pp. 506-515, Springer, Berlin, Germany, 2008.

[51] Z. Sunberg and J. Rogers, "A belief function distance metric for orderable sets," Information Fusion, vol. 14, no. 4, pp. 361-373, 2013.

[52] F. Cuzzolin, "A geometric approach to the theory of evidence," IEEE Transactions on Systems, Man, and Cybernetics, Part C (Applications and Reviews), vol. 38, no. 4, pp. 522-534, 2008.

[53] Y. Tang, D. Zhou, and F. Chan, "An extension to dDeng's entropy in the open world assumption with an application in sensor dData fusion," Sensors, vol. 18, no. 6, p. 1902, 2018.

[54] W. Jiang, Y. Luo, X.-Y. Qin, and J. Zhan, "An improved method to rank generalized fuzzy numbers with different left heights and right heights," Journal of Intelligent \& Fuzzy Systems, vol. 28, no. 5, pp. 2343-2355, 2015.

[55] R. R. Yager, "Jeffrey's rule of conditioning with various forms for uncertainty," Information Fusion, vol. 26, pp. 136-143, 2015.

[56] Y. Li and Y. Deng, "Generalized ordered propositions fusion based on belief entropy," International Journal of Computers, Communications \& Control, vol. 13, no. 5, 2015.

[57] D. Zhou, Y. Tang, and W. Jiang, "A modified belief entropy in dempster-shafer framework,” PLoS One, vol. 12, no. 5, 2017.

[58] Q. Pan, D. Zhou, Y. Tang, X. Li, and J. Huang, "A novel belief entropy for measuring uncertainty in dempster-shafer evidence theory framework based on plausibility transformation and weighted hartley entropy," Entropy, vol. 21, no. 2, p. 163, 2019.

[59] C. E. Shannon, "A mathematical theory of communication," ACM SIGMOBILE Mobile Computing and Communications Review, vol. 5, no. 1, pp. 3-55, 2001.

[60] D. Dubois and H. Prade, "A note on measures of specificity for fuzzy sets," International Journal of General Systems, vol. 10, no. 4, pp. 279-283, 1985.

[61] R. R. Yager, "Entropy and specificity in a mathematical theory of evidence," International Journal of General Systems, vol. 9, no. 4, pp. 249-260, 1983.

[62] G. J. Klir and A. Ramer, "Uncertainty in the dempster-shafer theory: a critical re-examination," International Journal of General Systems, vol. 18, no. 2, pp. 155-166, 1990.

[63] T. George and N. R. Pal, "Quantification of conflict in dempster-shafer framework: a new approach," International Journal of General Systems, vol. 24, no. 4, pp. 407-423, 1996.

[64] D. Maluf, "Monotonicity of entropy computations in belief functions," Intelligent Data Analysis, vol. 1, no. 1-4, pp. 207-213, 1997.

[65] G. J. Klir and M. J. Wierman, Uncertainty-based Information: Elements of Generalized Information Theory, Springer, Berlin, Germany, 1999.

[66] Y. Deng, "Deng entropy," Chaos, Solitons \& Fractals, vol. 91, pp. 549-553, 2016.
[67] W. Jiang, B. Wei, C. Xie, and D. Zhou, "An evidential sensor fusion method in fault diagnosis," Advances in Mechanical Engineering, vol. 8, no. 3, pp. 1-7, 2016.

[68] K. Yuan, F. Xiao, L. Fei, B. Kang, and Y. Deng, "Modeling sensor reliability in fault diagnosis based on evidence theory," Sensors, vol. 16, no. 1, p. 113, 2016.

[69] Y. Tang, D. Zhou, Z. He, and S. Xu, "An improved belief entropy-based uncertainty management approach for sensor data fusion," International Journal of Distributed Sensor Networks, vol. 13, no. 7, 2017.

[70] X. Fan and M. J. Zuo, "Fault diagnosis of machines based on D-S evidence theory. Part 1: D-S evidence theory and its improvement," Pattern Recognition Letters, vol. 27, no. 5, pp. 366-376, 2006. 